\title{
The Surgical Management of Traumatic Lower Cervical Spondylolisthesis with Posterior Percutaneous Pedicle Screw Fixation
}

\author{
Peng Luo, Wen-Fei Ni, Yao-Sen Wu, Ai-Min Wu, Xiang-Yang Wang, Hua-Zi Xu, Yong-Long Chi \\ Department of Spinal Surgery, Second Affiliated Hospital of Wenzhou Medical University, Wenzhou, China
}

We reported a technical report of traumatic lower cervical spondylolisthesisca used by bilateral pedicle fracture, without neurological compression. The patient was treated with the minimally invasive technique of percutaneous pedicle screw fixation. Fracture healing and normal cervical motion were confirmed by plain films and physical examinations onthe18-monthpostoperatively. The technique of percutaneous pedicle screw fixation might be an alternative strategy for the treatment of traumatic lower cervical spondylolisthesis with pedicle fracture.

Keywords: Cervical vertebrae; Spondylolisthesis; Pedicle screw

\section{Introduction}

Traumatic spondylolisthesis is uncommon in the cervical spine. The previous reports of traumatic cervical spondylolisthesis, mainly occurred at the axis that is widely recognized as "hangman's fracture" [1-4]. However, traumatic spondylolisthesis of the lower cervical spine is rare and only a few cases have been reported [5-8]. The purpose of this paper was to introduce a surgical method to manage the traumatic spondylolsthesis of the lower cervical spine.

\section{Technical Note}

\section{History and examination}

A 35-year-old woman who suffered from a motor vehicle accident, was initially sent to the orthopedics department at the Second Affiliated Hospital of Wenzhou Medical
College. The patient complained of severe neck pain with slight numbness in the ring and little fingers, and limited motion of the neck. Physical examination showed apparent tenderness of the seventh cervical spinous process. The neck pain became more severe in the positions of flexion, extension and lateral bending . Neurological examination demonstrated that sensory perception of the ring and little fingers was diminished, and normal motor function was limited. Lateral X-ray film and computed tomography (CT) scans of the cervical spine revealed that pedicles of the seventh cervical vertebra were fractured, and the seventh cervical vertebra had slipped forward. The integrity of facet joints was intact (Fig. 1A, B). No spinal cord compression, edema or hemorrhage was seen on magnetic resonance images (Fig. 1C). Primary management at our institution included axial traction via Gardner-Wells calipers for 2 days. Lateral radiograph confirmed a near-anatomic reduction of the fracture. The

Received Sep 18, 2014; Revised Sep 26, 2014; Accepted Oct 16, 2014

Corresponding author: Yong-Long Chi

Department of Spinal Surgery, Second Affiliated Hospital of Wenzhou Medical University,

109\# XueYuan Xi Road, Wenzhou, Zhejiang, China

Tel: +86-577-88002814, Fax: +86-577-88002823, E-mail: spinechi@sina.com 
surgical procedure was subsequently conducted .

\section{Operation}

After generalanesthesia, the patient was positioned prone on the operating table. Two small rolls were put under the shoulders to keep the neck slightly flexed. The head was placed in a U-type tractor and the shoulders were pulled caudally by heavy bandages to obtain a clear intraoperative lateral radiographic image of the seventh cervical spine. Spinal somatosensory evoked potential was monitored throughout the operation to monitor the physiological changes of spinal cord and nerves. Preoperatively, the bilateral skin entry point was measured in the axial plane of the CT scan, (about $3 \mathrm{~cm}$ relevant to the midline) (Fig. 1B). The position of skin entry point was the intersection point of the extension line of the seventh cervical pedicle axis with the posterior skin of the neck. The lateral angulation of the pedicle screw relative to the midline was also measured bilaterally on the same scan (approximately $30^{\circ}$ ) (Fig. 1B). According to the measured parameters, a $5 \mathrm{~mm}$ initial incision was made, about 3 $\mathrm{cm}$ from the midline at the level of the seventh cervical pedicle. A $1.2 \mathrm{~mm} \mathrm{~K}$-wire with a sharp tip was inserted and adjusted to reach the entry point (the midpoint of the lateral border of the pedicleon anteroposterior [AP] view) with fluoroscopic assistance. The procedure was repeated on the other side. We adjusted the position of the K-wires until they reached an angle of approximately $30^{\circ}$ lateral to the sagittal plane on an AP image, and paralleled the upper end-plate of the seventh cervical vertebra on a lateral view (Fig. 2A, B). The K-wires were then implanted into the seventh cervical pedicle across the fracture line with a power-drill, reaching the anterior cortex of the vertebral under fluoroscopic guidance (Fig. 2C). The depth of penetration was determined by the depth gauge, when the position of the K-wire was satisfactory. The guide tube (5.8 $\mathrm{mm}$ outer diameter, $1.2 \mathrm{~mm}$ inner diameter) was inserted over the K-wire. A protection sleeve, with a $7 \mathrm{~mm}$ outer diameter and $6.1 \mathrm{~mm}$ inner diameter, was advanced along the guide tube. The guide tube was subsequently withdrawn. A recess for the screw head was made using the cannulated drill bit sliding over the $\mathrm{K}$-wire. Care must be taken to avoid advancing the K-wire further while drilling. A $3.5 \mathrm{~mm}$ partially-threaded self-tapping cannulated screw was then placed over the K-wire inside the protection sleeve (Fig. 2D). Once the screw was well positioned, the K-wire and sleeve were removed simultaneously. The same approach was taken at the opposite side (Fig. 2E). Closure of the wound was performed with one stitch after checking for hemostasis.

\section{Postoperative course}

There were no operation-related complications during the postoperative period. The patient was discharged
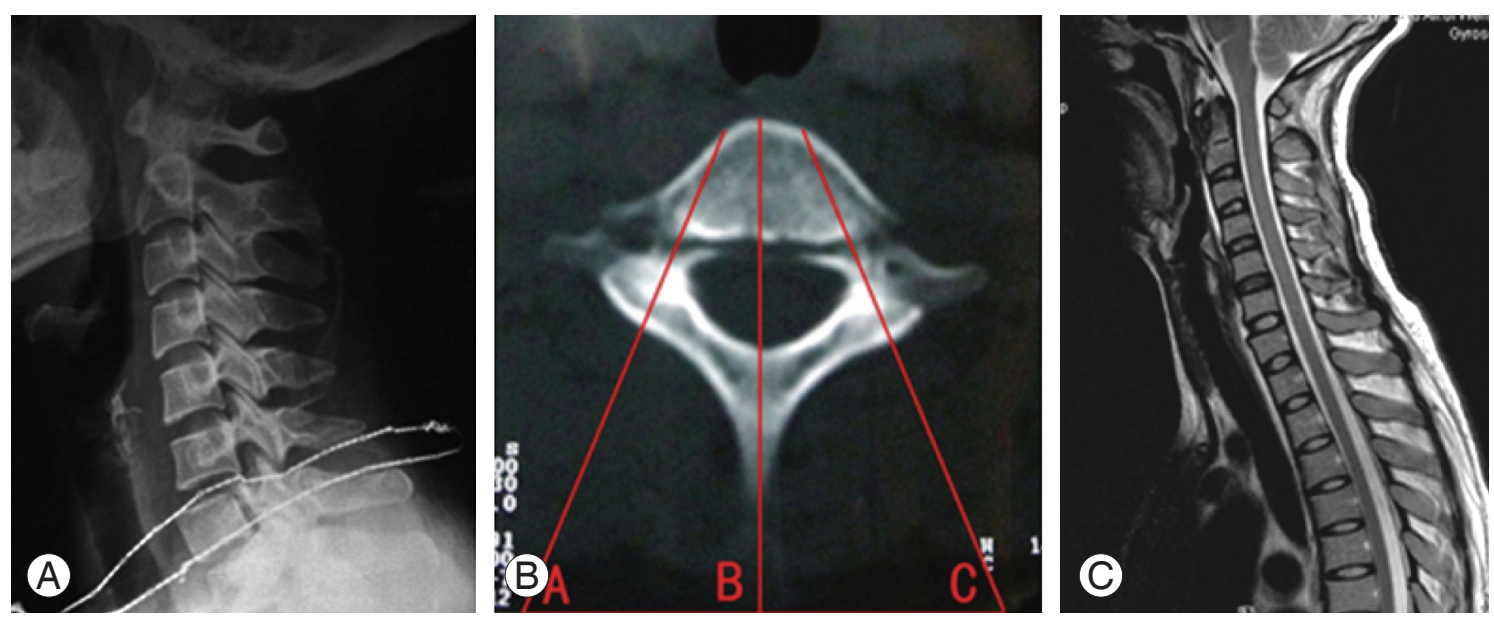

Fig. 1. (A) Plain lateral radiogram showing traumatic spondylolisthesis of C7, the seventh cervical vertebra was displaced forward relevant to its posterior elements. (B) Computed tomography image showing both sides of the pedicles of the seventh cervical vertebra fractured. The $\mathrm{A}$ and $\mathrm{C}$ were the points for skin puncture, the distance between the skin entry point to the midline of neck was about $3 \mathrm{~cm}$. The bilateral entry angle was $30^{\circ}$. (C) Magnetic resonance image showing no spinal cord compression, edema or hemorrhage. 

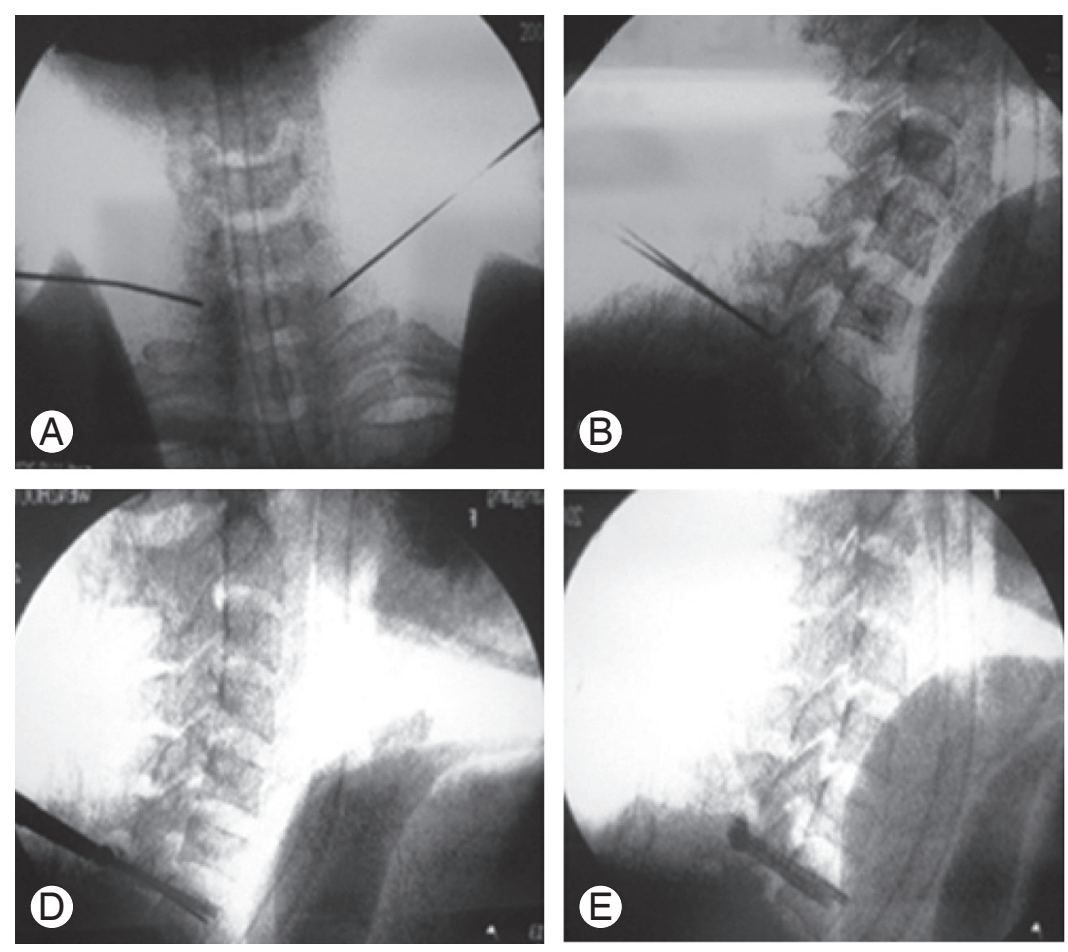

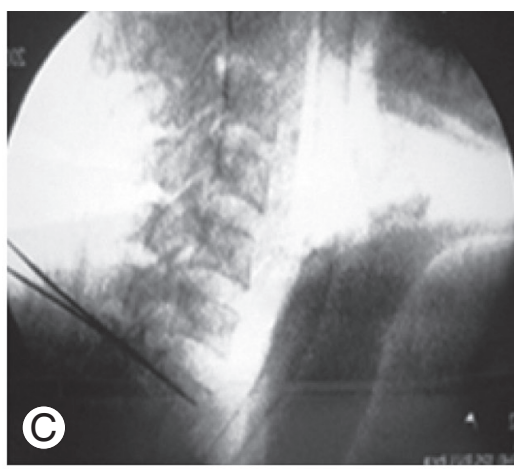

Fig. 2. (A) The K-wires were placed at the right position of the entry point on the anteroposterior view. (B) The K-wires were placed at the right position of entry point, paralleling the upper end-plate of the seventh cervical vertebra on the lateral view. (C) The K-wires were drilled into the seventh cervical pedicle across the fractures until they reached the anterior cortex of the vertebra. (D) The screw was tightened and the threads of screw were confirmed to cross the fracture line. (E) The final position of the screws.
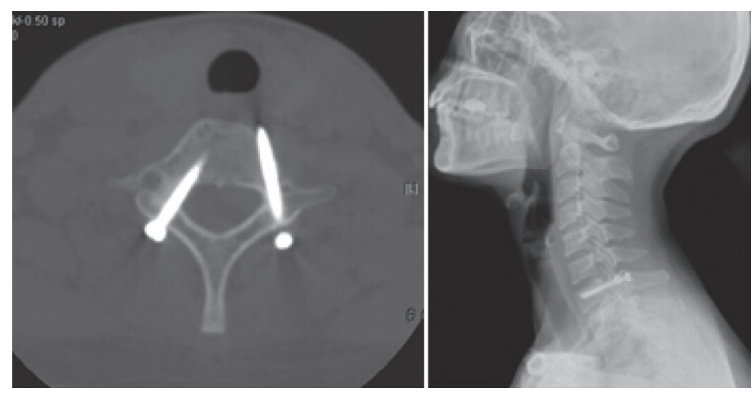

Fig. 3. Eighteen months postoperatively, computed tomography and lateral cervical $X$-rays showing solid union of the fracture. The motions of neck were normal.

from the hospital 4 days postoperatively, and a soft collar was placed for 8 weeks. She was followed-up at 18 months, with lateral cervical X-rays and CT that showed solid union of fractures of the seventh cervical vertebra. No pain and neurologic deficit was observed. The neck motions were normal (Fig. 3).

\section{Discussion}

Spondylolysis of the cervical spine is rarer than lumbar spondylolysis. The reported cases of cervical spondylolisthesis were mostly congenital in origin [9]. Very few cases of traumatic lower cervical spondylolisthesis have been reported [5-7]. According to a report by Meyer and Heim [10], of the 1,484 patients with cervical spine injuries between 1972 and 1986, there was only 1case with bilateral pedicle fracture of the lower cervical spine. Allen et al. [11] thought this pattern of injury was consistent with a high-grade compression-extension mechanism. Our patient had bilateral pedicle fracture with anterior slippage of the vertebral body that was compatible with stage 3 compressive extension according to the classification of Allen et al. [11]. The patients' mild neurological symptoms might be explained by the particular pattern of fracture. With integrity of the posterior elements, the diameter of the spinal canal was increased by the spondylolisthesis of anterior vertebral bodies. Many authors [12-14] believe that since the etiology of cervical spondylolisthesis is congenital in nature, conservative treatment for majority of patients will be successful. Surgery should be considered if the cervical spondylolisthesis was caused by an acute fracture, with segmental instability or spinal cord involvement. Dunn [5] reported a patient with bilateral pedicle fracture at the seventh cervical vertebrae with a bilateral facet dislocation who was treated by posterior fixation. The patient had a solid fusion without any neurologic deficit one year after the operation. Rovin et al. [7] described another case of traumatic cervical spondylolisthesis with the sixth cervicalpedicles and articular 
process fractures; he used the anterior approach for treatment and stable fusion was achieved 3 months postoperatively. Menku et al. [8] reported a successful result from a one-stage anterior combined with posterior approach, for managing a traumatic cervical spondylolisthesis.

Magerl [15] first describe percutaneous pedicle screws in conjunction with an external fixator for lower thoracic and lumbar disorders, in 1982. Minimally invasive percutaneous pedicle screws osteosynthesis has currently become a alternative method for treating lumbar vertebra fracture. However, there is no published report of this technique for the treatment of cervical pathologies. This article might be the first report of surgical management of traumatic cervical spondylolisthesis with percutaneous pedicle screw fixation. The minimally invasive percutaneous approach allows a maximum reduction in surgical morbidity and rapid recovery to basal functional status [16]. Direct screw fixation of the pars interarticularis allowed for preservation of the neighboring motion segments, which are thought to be a "physiological operation" described by Judet et al. [17]. Additionally, the application of threaded lag screws in this case could join both sides of the fracture line and theoretically promote healing. Johnson and Wang [18] thought that lag screw fixation might be the ideal instrumentation strategy for bilateral pedicle. The CT at the final follow-up showed union of the bilateral pedicle; the position of the screws appeared slightly misplaced. The patient did not present any neurological syndrome. We recommended that application of 3-dimensional navigation technology would be helpful for the described minimally invasive procedure.

This technique is not appropriate for all patients with traumatic lower cervical spondylolisthesis. Anterior decompression procedure is not required in patients without intervertebral disk injury and spinal cord compression. Moreover, no fracture or dislocation of the facets was seen in our patient. It was possible for lag screws to be percutaneously placed into the anterior fracture fragment in this unique pattern of cervical spondylolisthesis. Nevertheless, percutaneous pedicle screw fixation, used for treating cervical spondylolisthesis, has a much higher risk with the smaller diameter of cervical pedicle, than when used to treat lumbar disorders. Preoperative CT or magnetic resonance imaging of the cervical region should be carefully interpreted to increase the accuracy of pedicle screws.

\section{Conflict of Interest}

No potential conflict of interest relevant to this article was reported.

\section{References}

1. Francis WR, Fielding JW, Hawkins RJ, Pepin J, Hensinger R. Traumatic spondylolisthesis of the axis. J Bone Joint Surg Br 1981;63:313-8.

2. Kocis J, Wendsche P, Visna P, Muzik V, Hart R. Traumatic spondylolisthesis of the axis. Acta Chir Orthop Traumatol Cech 2003;70:214-8.

3. Boullosa JL, Colli BO, Carlotti CG Jr, Tanaka K, dos Santos MB. Surgical management of axis' traumatic spondylolisthesis (Hangman's fracture). Arq Neuropsiquiatr 2004;62:821-6.

4. Chittiboina P, Wylen E, Ogden A, Mukherjee DP, Vannemreddy P, Nanda A. Traumatic spondylolisthesis of the axis: a biomechanical comparison of clinically relevant anterior and posterior fusion techniques. J Neurosurg Spine 2009;11:379-87.

5. Dunn EJ. Fracture-dislocation of the posterior elements of $\mathrm{C} 7$ associated with bilateral pedicle fractures of C7: a case report. J Trauma 1974;14:527-36.

6. Byun HS, Patel PP. Unusual fracture/dislocation of the lower cervical spine: case report. Neurosurgery 1984;14:76-7.

7. Rovin RA, Brown JT, Meyer PR Jr. Traumatic spondylolisthesis of the lower cervical spine: case report. Neurosurgery 1992;30:124-7.

8. Menku A, Kurtsoy A, Tucer B, Oktem IS, Akdemir H. The surgical management of traumatic C6-C7 spondyloptosis in a patient without neurological deficits. Minim Invasive Neurosurg 2004;47:242-4.

9. Akay KM, Ersahin Y, Tabur E. Cervical spondyloptosis: a case report. Minim Invasive Neurosurg 2002;45: 169-72.

10. Meyer PR Jr, Heim S. Surgical stabilization of the cervical spine. In: Meyer PR Jr, editor. Surgery of spine trauma. New York: Chuchill-Livingstone; 1989. p.397-523.

11. Allen BL Jr, Ferguson RL, Lehmann TR, O’Brien RP. A mechanistic classification of closed, indirect fractures and dislocations of the lower cervical spine. Spine (Phila Pa 1976) 1982;7:1-27.

12. Hinton MA, Harris MB, King AG. Cervical spon- 
dylolysis. Report of two cases. Spine (Phila Pa 1976) 1993;18:1369-72.

13. Jones DN, Sage MR. Bilateral spondylolysis and associated dysplasia of C6. Australas Radiol 1992;36:2601.

14. Poggi JJ, Martinez S, Hardaker WT Jr, Richardson WJ. Cervical spondylolysis. J Spinal Disord 1992;5: 349-56.

15. Magerl F. External skeletal fixation of the lower thoracic andthe lumbar spine. In: Uhtoff $\mathrm{HK}$, editor. Current concepts of external fixation of fractures. Berlin: Springer-Verlag; 1982. p.353-66.
16. Ringel F, Stoffel M, Stuer C, Meyer B. Minimally invasive transmuscular pedicle screw fixation of the thoracic and lumbar spine. Neurosurgery 2006;59: ONS361-6.

17. Judet R, Roy-Camille R, Saillant G. Actualites de chirurgie orthopedique de l'Hospital Raymond-Poincare. In: Judet R, editor. Fractures du rachis cervical. 8th ed. Paris: Masson; 1970. p.174-95.

18. Johnson JN, Wang MY. Stress fracture of the lumbar pedicle bilaterally: surgical repair using a percutaneous minimally invasive technique. J Neurosurg Spine 2009;11:724-8. 